\title{
Перспективы применения
} технологий BIG DATA

\section{в атомной энергетике Украины}

Ястребенецкий М. А.

Государственное предприятие «Государственный научно-технический центр по ядерной и радиационной безопасности», г. Харьков, Украина ORCID: https://orcid.org/0000-0002-3662-9519

Дыбач А. М.

Государственное предприятие «Государственный научно-технический центр по ядерной и радиационной безопасности», г. Киев, Украина ORCID: https://orcid.org/0000-0002-1807-8514

Big data - термин, используемый по отношению к множеству разнообразных данных большого объема, требующих высокой скорости обработки. Оперирование с Big data включает в себя не только собственно данные, но и сбор данных, передачу, хранение, обработку (преобразование, вычисление, анализ), визуализацию и использование информации в различных направлениях человеческой деятельности. Возникновение Big data является одним из шагов к оцифровке всей информации, используемой в различных сферах деятельности.

В последние годы за рубежом началось применение big data в ряде отраслей промышленности, включая атомную энергетику. Можно предположить, что применение Big data в ближайшие годы может стать следующим революционным внедрением цифровых технологий для АЭС. В статье приведены примеры использования big data в различных странах для создания новых блоков и совершенствования эксплуатации действующих блоков.

Направления использования Big data при эксплуатации энергоблоков АЭС Украины:

- при предполагаемом создании отраслевой системы технической диагностики систем, структур и компонентов АЭС;

- для совершенствования работ по продолжению эксплуатации энергоблоков в сверхпроектный срок;

- для повышения безопасности информации благодаря использованию основанной на обработке Big data: структурированной информации об отказах оборудования и инцидентах на различных АЭС, хранящейся в международных и национальных базах данных; информации, получаемой из беспилотников, в частности при послеаварийном мониторинге и др.

Ключевые слова: большие данные, атомная электростанция, эксплуатация, информация, базы данных, система диагностики.

$\mathrm{O}$ дним из самых новых и чрезвычайно быстро развивающихся направлений в информационных технологиях являются Big data (большие данные), возникновение которого относится к 2008 г. [1]. Существует множество определений понятия Big data, более того, уже опубликованы работы, посвященные анализу и сопоставлению этих определений (например, [2]). Ограничимся двумя определениями.

Согласно [3], Big data - это термин, используемый по отношению к изучению и применению множеств данных, которые такие большие и сложные, что применение традиционного программного обеспечения для их обработки не позволяет справиться с этой задачей.

Согласно [4], Big data означает данные большего размера, которые можно легко обработать традиционными средствами, например такими, как реляционная база данных, без применения дорогостоящего специализированного программного обеспечения.

Big data характеризуется тремя $\boldsymbol{V}$ :

- Volume - большим объемом обрабатываемых и хранимых данных;

- Velocity - скоростью, когда требуется быстрые получение, обработка информации и демонстрация результатов обработки;

- Variety - разнообразием, когда необходима обработка различных типов данных, например, числовых, текстовых, изображений, видео, аудио.

В дальнейшем появились интерпретации с «четырьмя $\mathbf{V}$ » (добавлялась veracity - достоверность), «Пятью V» (в этом варианте прибавляли viability - жизнеспособность, и value - ценность, понимая под этим важность экономической целесообразности обработки соответствующих объёмов в соответствующих условиях), а также с прибавлением variability - переменчивость, и visualization.

Оперирование с Big data включает в себя не только собственно данные, но и сбор данных, передачу, хранение, обработку (преобразование, вычисление, анализ), визуализацию и использование информации в различных направлениях человеческой деятельности.

Возникновение Big data является одним из шагов к глобальной оцифровке всей информации, используемой в различных сферах деятельности. Одним из первых применений Big data являлось обработка потока посещений визитерами вебсайтов крупнейших корпораций для управления маркетингом - анализа данных о потребителях, включая историю их покупок и детальную информацию о каждой покупке, прогнозирования поведения потребителей. В настоящее время поток информации о Big data захлестывает интернет, опубликованы разнообразные книги, начиная от двухтомного издания [5] до книг общеобразовательного уровня [6].

Помимо традиционных методов статистического анализа (например, регрессионного анализа), в Big data используются ряд новых методов, как обработка естественного языка с интеграцией разнородных данных из различных источников, искусственные нейронные сети и др. Для использования этих методов разработан ряд новых программных и аппаратно-программных продуктов - нереляционная система управления базами данных NoSQL - Not Only Structured Query Language, Oracle Big Data Application и др.

В последние годы за рубежом началось применение big data в ряде отраслей промышленности, включая атомную энергетику. Из всех технологий, используемых в атомной энергетике, информационные технологии являются наиболее быстро меняющимися. Основу парка АЭС во всем 
мире составляют энергоблоки, выполненные по технологиям 70-90 годов прошлого столетия, сроки эксплуатации этих энергоблоков -30 и более лет. За время эксплуатации изменения в технологическом процессе не имеют глобального характера. В то же время изменения в информационных технологиях привели к полной замене аналоговой техники на цифровую в информационных и управляющих системах энергоблоков АЭС, управляющих технологическим процессом непосредственно или с участием оперативного персонала энергоблоков. Это было первое революционное внедрение цифровых технологий для АЭС. Отметим, что и цифровая техника для управления АЭС уже сменила одно или более поколений.

Можно предположить, что применение Big data в ближайшие годы может стать следующим революционным внедрением цифровых технологий для АЭС. Так в США в 2017 г. и 2018 г. Ohio State University (prof. C.Smidts) проводил конференции по применению big data для АЭС ([7], [8]). Уже в 2019 г. на 11-й конференции ядерного общества США “Nuclear Plants Instrumentation, Control and Human-Machine Interface Technologies" впервые имела место техническая сессия, посвященная анализу big data для АЭС (председатель сессии - M.Khafizov). Отметим, что эта конференция является наиболее представительной и авторитетной в мире по автоматике АЭС, а с 3-й по 9-ю этих конференций заслушивались доклады ГНТЦ ЯРБ.

Пожалуй, наиболее эффективным является комплексное применение big data для всех этапов жизненного цикла при разработке новых энергоблоков, когда собираются и используются данные по их сооружению (проектированию, строительству, испытаниям, комплектации и др.), обновляемые в реальном времени. Такие работы начали проводить Westinghouse, «Росатомэнерго» и др. при создании так называемых цифровых двойников АЭС (иное название - цифровая АЭС) и отдельных видов оборудования. "General Electric" разработал цифровой двойник турбины, включающий архив показаний и состояния машины, анализ всех машин этой модели. "General Electric" предлагает цифровой продукт "Predix" для потребностей в промышленности (не только в атомной энергетике) для быстрого анализа больших данных при сохранении требований кибербезопасности на облачной платформе.”General Electric" уже создал большое количество цифровых двойников (см. $[9,10])$.

Создание указанных выше моделей является чрезвычайно перспективной проблемой, но далее рассмотрим более частные задачи, применимые к потребностям и возможностям Украины в настоящее время.

Украина не является страной, разрабатывающей энергоблоки АЭС, здесь выпускаются отдельные системы (например, информационные и управляющие) и ряд видов оборудования (турбины, насосы). Сооружение новых энергоблоков после пуска энергоблоков Ровенской и Хмельницкой АЭС в 2004 г. застопорилось по ряду причин. В то же время в Украине функционируют 15 энергоблоков типа ВВЭР, составляющие основу выработки электроэнергии государства, и их эксплуатация является насущной проблемой. Из них 11 энергоблоков ВВЭР-1000 одной модели - В-320. Это дает возможность оперировать большим объемом однородной статистической информации. Областью применения Big data может быть совершенствование эксплуатации и повышение безопасности действующих энергоблоков.
Направления использования Big data при эксплуатации энергоблоков

Отраслевая система технической диагностики систем, структур и компонентов АЭС

В основе этого направления лежит оперативное получение, обработка и визуализация информации от датчиков о состоянии структур, систем и элементов АЭС.

Более 10 лет назад харьковская корпорация «Техностандарт» выступила с предложением о разработке такой системы. Руководителем работы в этой организации был И.И. Иванисов, до того много лет работавший заместителем начальника цеха тепловой автоматики и измерений Запорожской АЭС, а потом - заместителем директора этой АЭС (сейчас И.И. Иванисов - начальник управления строительства на площадке АЭС «Куданкулам» Индия). ГНТЦ ЯРБ был приглашен к участию в создании этой системы, но ее выполнение затормозилось уже на начальной стадии.

В рамках мероприятий по выполнению работ в соответствии с «Комплексной (сводной) программой повышения безопасности АЭС Украины», Северодонецкое НПО «Импульс» выполнил разработку и внедрение на энергоблоках АЭС комплексной системы диагностики оборудования реакторной установки (работы по бл. 5, 6 ОП ЗАЭС будут выполнены в ППР-2019, 2020).

В настоящее время с предложением по созданию и внедрению комплекса технической диагностики в ГП «НАЭК «Энергоатом» выступил научно-технический центр НАЭК. Предварительное обсуждение принципов создания такой системы с автором предложений В.Н. Колесником (много лет возглавлявшим ЦТАИ Ровенской АЭС) показало ее перспективность. Следует отметить, что работы в этой области по заданию ГП НАЭК «Энергоатом» выполнял ИПМЭ им. Г.Е. Пухова совместно с ГП «КБ «Атомприбор». УПомянутый институт НАНУ разработал проект Концепции построения многоуровневой отраслевой системы мониторинга, диагностирования и прогнозирования технического состояния АЭС Украины [11].

За рубежом комплексы технической диагностики систем, структур и компонентов АЭС активно развиваются. Например, Департаментом энергетики CША (U.S. DOE) peaлизован пилотный проект по применению Big data для АЭС с целью идентификации и прогнозирования возникновения вибрации отдельных типов арматур, выявления и устранения ее коренной причины [12]. Ведутся работы в этом направлении и компанией Westinghouse, которая разработала платформу для мониторинга состояния оборудования на основании Big data [13]. В начале 2018 г. Корея [14] анонсировала создание своей первой Big data системы предварительной диагностики, анализа и оценки оборудования АЭС. Система должна иметь связь с 24 энергоблоками страны через передачу в реальном времени данных о 16000 единиц оборудования (турбины, питательные насосы и др.). Разработку системы предполагается закончить в 2020 г. Стоимость разработки - 36 млн. \$. К августу 2018 г. охвачено 240 единиц оборудования через беспроводные датчики.

Рассмотрим соответствие задачи создания отраслевой системы технической диагностики АЭС Украины принципам $3 \mathrm{~V}$ :

- объем данных о состоянии оборудования даже одного блока весьма велик, а 15 энергоблоков четырех АЭС - тем более;

- скорость обработки информации нужна для оперативного предотвращения отказов оборудования, 
особенно - приводящих к простоям или снижению мощности энергоблоков;

- разнообразие представляемой информации (например, представление изображений) позволит решать ряд новых задач технической диагностики, в частности выявление скрытых отказов и скрытых закономерностей в поведении систем, структур и компонентов АЭС.

Продолжение эксплуатации энергоблоков в сверхпроектный срок

Это направление чрезвычайно актуально во всем мире, учитывая старение парка действующих энергоблоков, и в Украине в частности. Бесспорно, совершенствование диагностики, о которой говорилось выше, является важным фактором, способствующим продолжению эксплуатации АЭС. Приведем примеры применения big data для задач продолжения эксплуатации энергоблоков.

Широко известная организация в США - «Electrical Power Research Institute (EPRI)» разработала программный продукт, предназначенный для прогнозирования и менеджмента срока эксплуатации парка АЭС и названный «FleetWide Prognostics and Health Management (FW-PHM)» [15]. В этом продукте предусмотрены алгоритмы он-лайн автоматической диагностики, прогнозирование состояния оборудования, анализа остаточного срока службы, распознавания образов и др. Компания «Rolls-Royce», имеющая значительный опыт работ по применению Big data в авиации, использует эти знания для анализа эксплуатационной информации в атомной энергетике [16].

Необходимым требованием для продления срока эксплуатации является подтверждение возможности такого продолжения регулирующим органом, для чего согласно международным рекомендациям [17], нормам и правилам Украины [18,19] необходима разработка соответствующей документации. Так, требуемый регулирующим органом отчет по периодической переоценке безопасности должен содержать анализ 14 факторов безопасности (ФБ)- аспектов эксплуатации энергоблока, влияние которых на безопасность энергоблока нужно рассмотреть при проведении периодической оценки безопасности.

Для факторов безопасности ФБ2 «Текущее состояние систем и элементов», ФБЗ «Квалификация оборудования», ФБ4 «Старение сооружений, систем и элементов» характерны большие массивы собираемых данных по системам, оборудованию, конструкциям, зданиям и сооружениям, влияющим на безопасность АЭС, которые требуют обработки и анализа для подтверждения соответствия регулирующим требованиям. Так же чрезвычайно интересным представляется сравнение данных по одному энергоблоку с данными для однотипных элементов других энергоблоков, что позволит выявить как общие тенденции, так и проблемные моменты на отдельных энергоблоках, обусловленные спецификой эксплуатационной практики и проведения технического обслуживания и ремонтов.

Рассмотрим соответствие задачи обработки данных по управлению старением принципам $3 \mathrm{~V}$ :

- большой объем анализируемых данных, необходимых для доказательства соответствия требованиям к продлению срока эксплуатации для каждого энергоблока;

- скорость обработки информации существенна потому, что весьма велики затраты времени, необходимые для подготовки документации;

- разнообразие подготавливаемой документации очевидна уже из названий факторов безопасности - разделов отчета по периодической переоценке безопасности.
Применение Big data, при продлении срока эксплуатации, может так же помочь при подтверждении перед общественностью правильности этой акции. Этот вопрос рассмотрен в [20] - Корея имеет много стареющих реакторов и производится оценка проектов продления сроков их эксплуатации. Big data используется специально для измерения общественного мнения, информирования населения и взаимодействия с ним.

Перспективные направления использования Big data для повышения безопасности АЭС

Отраслевая система технической диагностики является дополнительным источником получения детальной информации о состоянии систем, структур и компонентов АЭС и прогнозируемой информации о состоянии энергоблока, что повышает надежность оборудования АЭС (например, за счет снижения числа скрытых отказов и перевода их в явные) и, следовательно, безопасность.

Повышению безопасности послужит также представление следующей информации, основанной на обработке Big data:

- данные противоаварийных тренировок оперативного персонала с полномасштабных тренажеров АЭС, а также данные по действиям оперативного персонала в режиме он-лайн;

- сгенерированные массивы расчетных данных по возможным метеоусловиям, характеристикам радиоактивного выброса, защитным мероприятиям с учетом их различных комбинаций и вероятностей для целей использования в аварийной готовности при принятии решений на краткосрочной фазе аварии [21];

- данные по технологическим параметрам и конфигурации оборудования энергоблока АЭС в режиме он-лайн;

- структурированной информации об отказах оборудования и инцидентах на различных АЭС, хранящейся в международных и национальных базах данных;

- информации, получаемой из беспилотников, в частности при послеаварийном мониторинге (см., например, [22]).

Представляется также интересным рассмотрение возможности использования Big data для анализа процесса регулирования ядерной и радиационной безопасности как задачи управления в замкнутой системе (см. [23]).

Что и кто нужны для применения big data для эксплуатации АЭС в Украине.

Необходимо участие специалистов АЭС и других организаций НАЭК «Энергоатом», Госатомрегулирования (включая ГНТЦ ЯРБ), институтов НАНУ, университетов в постановке задач для атомной энергетики с учетом возможностей Big data, включая разработку методов оценки получаемой информации. Требуется разработка или приобретение программного обеспечения с учетом обеспечения компьютерной безопасности. Необходима подготовка персонала для эксплуатации систем с применением Big data.

Задача применения Big data в атомной энергетике Украины облегчается наличием в Украине высококлассных специалистов по информационным технологиям и по математической статистике в университетах, Национальной Академии Наук, различных предприятиях. Накопленный опыт специалистов Украины по созданию и эксплуатации цифровой вычислительной техники для информационных и управляющих систем энергоблоков АЭС (причем не только АЭС Украины, но и для иных стран) даст возможность применить этот опыт и при использовании систем с Big data. 


\section{Благодарности}

- V. Melnikov, Ph.D, Prof. Applied Statistics of the University of Alabama (выпускнику кафедры «Техническая кибернетика» Харьковского Политехнического института), который в 2018 г. прочитал лекции по современным задачам математической статистики для сотрудников Харьковского филиала ГНТЦ ЯРБ и обратил их внимание на проблему Big data.

- Л. М. Любчику, д.т.н., проф., зав. кафедрой «Компьютерной математики и анализ данных» Национального технического университета «Харьковский политехнический институт» за обсуждение настоящей статьи.

\section{Список использованной литературы}

1. Mashey John R. Big Data and the Next Wave of InfraStress. Slides from invited talk. Usenix. Retrieved, 28 September 2016.

2. Gil Press. 12 Big Date Definitions. What's yours? - ULR: https:// www.forbes.com/sites/gilpress/2014/09/03/12-big-data-definitionswhats-yours

3. Big data. - ULR: https://en.wikipedia.org/wiki/Big_data

4. Tigani Jordan, Siddartha Naidu. Google Big Query Analytics. John Wiley \& Sons. 2014.

5. Segall Richard S., Cook Jeffrey S. Handbook of Research on Big Data Storage and Visualization Techniques (2 Volumes). IGI Global, 2018.

6. Wessler Michael. Big data Analytics for Dummies. John Wiley \& Sons. 2013

7. ULR: https://mae.osu.edu/events/2017/09/big-data-nuclear-powerplants-workshop.

8. ULR: https://bigdata.engineering.osu.edu/

9. Кудряшев К. Цифровая АЭС - это уже не фантастика. ULR: http://atomicexpert.com/page1080930.html

10. Плотонова О. Россия создает виртуальную АЭС. - ULR: http://atomicexpert.com/page2607825.html

11. Владимирский А. А., Мохор В. В. Плескач Б. Н., Киндрась А. Л. «Проект концепции построения многоуровневой отраслевой системы мониторинга, диагностирования и прогнозирования технического состояния АЭС Украины». Моделювання та інформаційні технології, 2017, вип. 79. с. 16-20.

12. Gruenwald T. Using "Big Data" to help understand NPP challenges. Advanced Censors and Instrumentation. October $31-$ November 1, 2018.

13. NA-0150. Westinghouse Electric Company. Nuclear Automation, Big Data Analytics. June 2015.

14. Jae-Min Cha, Junguk Shin, Choong-Sub Yeom. A Review on Applicability of Big Data Technology in Nuclear Power Plant: Focused on O\&M Phases. Transactions of the Korean Nuclear Society Spring Meeting. Jeju, Korea, 7-8 May 2015.

15. Rusaw R. Big data meets nuclear power. Nuclear Engineering International. 2014. - ULR: http://www.neimagazine.com/features/ featurebig-data-meets-nuclear-power-4272532

16. Bruce Power and Rolls-Royce sign multi-year cooperation agreement for plant life extension. ULR: http://www.brucepower.com/ rolls-royce-cooperation-agreement/

17. IAEA Safety Standard Series No. SSG-25. Periodic Safety Review of Nuclear Power Plants. Vienna, 2013.

18. НП 306.2.141-2008. Загальні положення безпеки атомних станцій. К.: Державний комітет ядерного регулювання України, 2008. $57 \mathrm{c}$

19. НП 306.2.099-2004. Загальні вимоги до продовження експлуатації енергоблоків АЕС у понадпроектний строк за результатами здійснення періодичної переоцінки безпеки. К.: Державний комітет ядерного регулювання України, 2004. $21 \mathrm{c}$.

20. Seungkook Roh. Big Data Analysis of Public Acceptance of Nuclear Power in Korea. Nuclear Engineering and Technology. 2017. V. 49, № 4. P.850-854.

21. Nuclear emergency response and Big Data technologies. Wolfgang Raskob and Stella Mцhrle. Karlsruhe Institute of Technology (KIT).

22. Саченко А. А., Кочан В. В., Харченко В. С., Ястребенецкий М. А., Фесенко Г. В., Яновский М. Э. Система послеаварийного мониторинга АЭС с использованием беспилотных летательных аппаратов: концепция, принципы построения. Ядерна та радіаційна безпека. 2017. № 1. С.24-29.

23. Ястребенецкий М. А. Регулирование ядерной и радиационной безопасности как задача системного анализа. Ядерна та радіаційна безпека. 2010. № 3. С.25-32.

\section{References}

1. Mashey, John R. (2016), "Big Data and the Next Wave of Infra Stress", Slides from Invited Talk, Usenix, Retrieved on 28 September 2016

2. Press, G. (2014), "12 Big Data Definitions. What's yours?", available at: https://www.forbes.com/sites/gilpress/2014/09/03/12-bigdata-definitions-whats-yours

3. "Big Data", available at: https://en.wikipedia.org/wiki/Big_data

4. Tigani, J., Siddhartha, N. (2014), "Google Big Query Analytics", John Wiley \& Sons.

5. Segall Richard S., Cook Jeffrey S. (2018), "Handbook of Research on Big Data Storage and Visualization Techniques (2 Volumes)", IGI Global.

6. Wessler, M. (2013), "Big Data Analytics for Dummies", John Wiley \& Sons, available at: https://mae.osu.edu/events/2017/09/bigdata-nuclear-power-plants- workshop.

7. Reference available at: https://mae.osu.edu/events/2017/09/bigdata-nuclear-power-plants- workshop.

8. Reference available at: https://bigdata.engineering.osu.edu/

9. Kudriashov, K., "Digital NPP Is No Longer a Fantasy" [Tsyfrovaia AES - eto uzhe ne fantastika], available at: http:// atomicexpert.com/page1080930.html (Rus).

10. Plotonova, O., "Russia Creates Virtual NPP" [Rossiia sozdaiot virtualnuiiu AES], available at: http://atomicexpert.com/page2607825. html (Rus).

11. Vladimirskii, A., Mokhor, V., Pleskach B., Kindras A., "Draft Concept of Constructing Multi-Layered Industry System for Monitoring, Diagnostics and Prediction of Technical State of Ukrainian NPPs" [Proekt kontseptsii postroieniia mnogourovnevoi otraslevoi sistemy monitoringa, diagnostirovaniia i prognozirovaniia tekhnicheskogo sostoianiia AES Ukrainy] (Rus). "Modelling and Information Technologies"

12. Gruenwald, T. (2018), "Using Big Data to Help Understand NPP Challenges. Advanced Censors and Instrumentation".

13. NA-0150 (2015), "Westinghouse Electric Company, Nuclear Automation, Big Data Analytics".

14. Jae-Min Cha, Junguk Shin, Choong-Sub Yeom (2015), "A Review on Applicability of Big Data Technology in Nuclear Power Plant: Focused on O\&M Phases", Transactions of the Korean Nuclear Society Spring Meeting, Jeju, Korea.

15. Rusaw, R. (2014), "Big Data Meets Nuclear Power. Nuclear Engineering International”, available at: http://www.neimagazine.com/ features/featurebig-data-meets-nuclear-power-4272532.

16. "Bruce Power and Rolls-Royce sign Multi-Year Cooperation Agreement for Plant Life Extension", available at: http://www. brucepower.com/rolls-royce-cooperation-agreement/

17. IAEA Safety Standard Series No. SSG-25 (2013), "Periodic Safety Review of Nuclear Power Plants", Vienna.

18. NP 306.2.141-2008 (2008), "General Safety Provisions for Nuclear Power Plants" [Zahalni polozhennia bezpeky atomnykh stantsii], Kyiv, SNRIU, 57 p. (Ukr).

19. NP 306.2.099-2004 (2004), "General Requirements for LongTerm Operation of NPP Units upon Results of Periodic Safety Review" [Zahalni vymohy do prodovzhennia ekspluatatsii enerhoblokiv AES u ponadproektnyi strok za rezultatamy zdiisnennia periodychnoi pereotsinky bezpeky], Kyiv, SNRIU, 21 p. (Ukr).

20. Seungkook, R. (2017), "Big Data Analysis of Public Acceptance of Nuclear Power in Korea", Nuclear Engineering and Technology, Volume 49, No. 4, pp. 850-854.

21. Raskob, W., Muhrle, S., "Nuclear Emergency Response and Big Data Technologies", Karlsruhe Institute of Technology (KIT).

22. Sachenko, A., Kochan, V., Kharchenko, V., Yastrebenetskyi, M., Fesenko, G., Yanovsky, M. (2017), "NPP Post-Accident Monitoring 
System Based on Unmanned Aircraft Vehicle: Concept, Design Principles" [Sistema posleavariinogo monitoring AES $\mathrm{s}$ ispolzovaniiem bespilotnykh letatelnykh apparatov: kontseptsiia, printsypy postroieniia], Nuclear and Radiation Safety, No. 1, pp. 2429 (Rus).

23. Yastrebenetskyi, M. (2010), "Nuclear and Radiation Safety Regulation as a Task of System Analysis" [Regulirovaniie yadernoi i radiatsionnoi bezopasnosti kak zadacha sistemnogo analiza], Nuclear and Radiation Safety, No. 3, pp. 25-32 (Rus).

\section{Перспективи застосування технологій BIG DATA в атомній енергетиці України}

\section{Ястребенецький М. О., Дибач О. М.}

Державне підприємство «Державний науково-технічний центр з ядерної та радіаційної безпеки», м Київ, м. Харків, Україна

Big data -термін, який використовується по відношенню до безлічі різноманітних даних великого обсягу, що вимагають високої швидкості обробки. Оперування з Big data включає в себе не тільки власне дані, а й збір даних, передачу, зберігання, обробку (перетворення, обчислення, аналіз), візуалізацію і використання інформації в різних напрямках людської діяльності. Виникнення Big data $\epsilon$ одним з кроків до оциф рування всієї інформації, використовуваної в різних сферах діяльності.

В останні роки за кордоном почалося застосування big data в ряді галузей промисловості, включаючи атомну енергетику. Можна припустити, що застосування Big data в найближчі роки може стати наступним революційним впровадженням цифрових технологій для AEC. у статті наведені приклади використання big data в різних країнах для створення нових блоків і вдосконалення експлуатації діючих блоків. Напрямки використання Big data при експлуатації енергоблоків АЕС України:

- при передбачуваному створенні галузевої системи технічної діагностики систем, структур і компонентів АEC,

- для вдосконалення робіт з впродовження експлуатації енергоблоків у понадпроектний термін;

- для підвищення безпеки завдяки використанню інформації, заснованої на обробці Big data: структурованої інформації про відмови обладнання і інциденти на різних AEC, що зберігається в міжнародних і національних базах даних; інформації, одержуваної з безпілотників, зокрема при післяаварійному моніторингута ін.

Ключові слова: великі дані, атомна електростанція експлуатація, інформація, бази даних, система діагностики.

\section{Prospects of Using Big Data Technologies in Nuclear Energy of Ukraine}

\section{Yastrebenetskyi M., Dybach 0.}

State Enterprise "State Scientific and Technical Center for Nuclear and Radiation Safety", Kharkiv, Kyiv, Ukraine

Big data is a term used for a wide variety of large data that require high processing speed. Big data handling includes not only the data, but also data collection, transmission, storage, processing (conversion, calculation, analysis), visualization and use of information in various areas of human activities. The emergence of Big data is one of the steps to digitizing all information used in different spheres.

In recent years, Big data are used in foreign countries in a number of industries, including nuclear energy. It can be assumed that the Big data use in the coming years may become the next revolutionary introduction of digital technologies for NPPs. The paper provides examples of using the Big data in various countries to create new units and improve operating units.

The areas of Big data use in the operation of Ukrainian NPPs are as follows:

- in intended creation of an industrial system for technical diagnostics of systems, structures and components of NPPs;

- for improving activities on long-term operation of power units;

- for improving safety becouse of using of information based on Big data: structured information on equipment failures and incidents at different NPPs stored in international and national databases; information obtained from drones, in particular in post-accident monitoring, etc.

Keywords: Big data, nuclear power plant, operation, information, database, diagnostic system. 